\title{
The impact of unsupervised regular walking on health: a sample of Turkish middle-aged and older adults
}

\author{
Orcin Telli Atalay • Uğur Cavlak
}

Received: 7 February 2011 / Accepted: 5 June 2011 / Published online: 22 June 2011

(C) European Group for Research into Elderly and Physical Activity (EGREPA) 2011

\begin{abstract}
Regular walking is one of the most recommended and popular physical activity worldwide. This study (1) detected the effects of unsupervised regular walking on physical and cognitive functioning, emotional status, and quality of life in middle-aged and older adults and (2) compared the results with inactive controls. Forty middleaged and older adults with a mean age of $56.30 \pm 4.85$ years (range 40-70) walking for at least 1 year, at least three times a week, and at least $45 \mathrm{~min}$ a day and 40 inactive participants with a mean age of $55.15 \pm 5.64$ years (range 40-70) participated. Before testing, sociodemographics of the participants were recorded. Body mass index and waist-hip ratio were calculated. The two groups were evaluated and compared in terms of physical functioning (handgrip strength, balance performance, cardio-vascular endurance, flexibility, muscular endurance and coordination), cognitive functioning (Mini Mental Test), emotional status (Beck Depression Inventory), and health related quality of life (CDC HRQOL4). The results obtained from this study showed that there were significant differences in terms of all outcome measurements between the groups $(p<0.05)$. The regular walking group had better scores in most parameters than the inactive controls. The results indicate that unsupervised regular walking improves health and is also a safe, cheap, and can easily be adapted into daily life. Therefore, it can be recommended to improve physical and cognitive functioning, emotional status, and quality of life of middle-aged and older adults.
\end{abstract}

\footnotetext{
O. T. Atalay $(\bowtie) \cdot U$. Cavlak

Pamukkale University,

School of Physical Therapy \& Rehabilitation,

Rektörlük Binasi, B Kati, Kinikli Kampüsü, Kinikli,

20070 Denizli, Turkey

e-mail: orcin_t@hotmail.com
}

Keywords Middle-aged · Older adults · Regular walking · Physical functioning $\cdot$ Cognitive function $\cdot$ Emotional status · Quality of life

\section{Introduction}

Average life period of people in Turkey is getting longer, but improving quality of life $(\mathrm{QoL})$ is more important than having a longer life. Many factors related with strength, endurance, and quality of life inclined to recede as a result of the changes in organism during growth and aging. The most important factor influencing physical capacity which is an important indicator of quality of life is modern lifestyle. Technological improvements and frequent use of transportation facilities, television, and computers restrict physical activity and decrease energy consumption. Energy consumption decreases more in older people with the restriction of physical activity as a result of aging. Physical inactivity caused by industry and modern lifestyle has negative effects on people at every age. Inactive lifestyle causes severe health problems like hypertension, obesity, muscle weakness, postural defects, diabetes and cardiovascular problems, restriction in pulmonary function, and gastrointestinal diseases in middle-aged and older people [1-3]. Although physical inactivity is a complex risk for unhealthy life developed countries, the studies concerning to change the course and reasons for inactivity problems are limited. According to WHO, about 1.9 million death and 19 million disability occur as a result of inactivity [4]. Since mid-1990s, the importance of moderate physical activity that lasts at least $30 \mathrm{~min}$ has been emphasized to communities [5]. Walking is one of the most recommended physical activities as it is easy and cheap and has lower risk of musculoskeletal injury which can be easily adapted to lifestyle. Many studies that were carried out in different 
countries showed that walking was also the most preferred activity because it is tolerated well and improved health and cardiovascular endurance [6-8]. According to the related literature, active people have improvements in health-related parameters by walking program $[3,5,7]$. There are strong evidences that support the health benefits of physical activity for the general population. Participating in moderate intensity physical activity (such as brisk walking) has been shown to reduce morbidity and mortality due to chronic disease and disability [9]. Walking is the most accessible physical activity for healthy life because it is an activity most people can do and is associated with a low injury rate [10]. Although there are many studies about the effects of treadmill walking program (supervised) on health-related parameters, there is not much data about the effects of regular walking in field (unsupervised). The primary aim of this study was to detect the effects of unsupervised regular walking on physical and mental health and quality of life in middle-aged and older adults comparing with inactive controls.

\section{Methods}

Study design and participants

Participants were reached and recruited through a local recreational and sports area (Camlik Mesire alani) in city of Denizli, Turkey. After informed consent was obtained, an experienced physical therapist and three final year physical therapy students started to test in the morning session at weekend. This is a comparative field study which was approved by Research Ethics Board of Medical Faculty at Pamukkale University in Denizli, Turkey. Written informed consent was obtained from the participants as approved by the committee. This study was supported by Pamukkale University Scientific Research Projects Foundation (grant no. 2008SBE003).

One hundred and seventy subjects, who met the inclusion criteria, were asked to participate in the study. However, $47.05 \%$ of the subjects just invited in the study accepted to participate. The sample size of 80 participants living in city of Denizli located in the western part of Turkey was studied (see Table 1). Forty middle-aged and older adults walking for at least 1 year and 40 inactive controls were evaluated. Each potential participant was screened for eligibility, including the following: The inclusion criteria for the walking group were:

1. Age $\geq 40$ years

2. Walking at least three times a week at least 45 min a day

3. Walking without any support

4. Having no neurological or orthopedic problem
The inclusion criteria for the controls were:

1. Age $\geq 40$ years

2. Presenting inactive lifestyle

3. Having no neurological or orthopedic problem

Participants who did not complete all the tests just needed for the study were excluded.

\section{Outcome measures}

Baseline measures, including participant demographics, physical properties, body mass index score, and waist-hip ratio score, were assessed and recorded before testing (see Tables 1 and 2). All tests were instructed using standard procedures, and all were based on objective measure or were self-reported.

\section{Physical functioning}

Physical functioning outcomes were well-known physical fitness field tests.

Handgrip test Upper extremity strength was assessed with handgrip strength test using a hydraulic dynamometer [11-13]. In this test, the participant was in sitting position on an unsupported chair with arms flexed next to trunk. The participant was asked to grip the dynamometer and squeeze as hard as he/she could do. The measurement was repeated three times and the average was recorded.

Balance assessment Both static and dynamic balance of each participant was measured. One-leg stance test [14] was used to measure static balance. The participant was asked to stand on his/her dominant leg with the other knee flexed 90. The shoes were off and his/her eyes were opened. The duration that participants kept their balance on this position was recorded in seconds. If there was not any loose in balance, the test was finished in 180th second. Dynamic balance was measured with sit and stand test which is a part of Senior Fitness Test [15]. The participants were asked to stand from a chair without any support and sit again as quickly as possible. This was repeated five times and the duration to do this test without any balance loose was recorded in second.

Cardiovascular endurance test This is a protocol for the 6-min test which is a common test especially used in geriatrics $[16,17]$. The walking distance of the participants in 6 min was recorded using a pedometer.

Flexibility The following tests were used to measure flexibility of the participants: (1) sit and reach [18] and (2) trunk lateral flexion [19]. 
Table 1 Demographics of the sample

\begin{tabular}{|c|c|c|c|c|}
\hline \multirow[t]{2}{*}{ Characteristics } & \multicolumn{2}{|c|}{ Regular walking group $(N=40)$} & \multicolumn{2}{|c|}{ Inactive controls $(N=40)$} \\
\hline & Number & $\%$ & Number & $\%$ \\
\hline \multicolumn{5}{|l|}{ Sex } \\
\hline $\mathrm{F} / \mathrm{M}$ & $18 / 22$ & $45 / 55$ & $21 / 19$ & $52.2 / 47.5$ \\
\hline \multicolumn{5}{|l|}{ Occupation } \\
\hline Housewife & 4 & 10 & 16 & 40 \\
\hline Registered official & 36 & 90 & 24 & 60 \\
\hline \multicolumn{5}{|l|}{ Education level } \\
\hline University level & 37 & 92.5 & 18 & 45 \\
\hline$<8$ years & 3 & 7.5 & 22 & 55 \\
\hline \multicolumn{5}{|l|}{ Smoking } \\
\hline Yes/no & $7 / 20$ & $17.5 / 50$ & $11 / 15$ & $27.5 / 32.5$ \\
\hline \multicolumn{5}{|c|}{ Operation at least once } \\
\hline Yes/no & $19 / 21$ & $47.5 / 52.5$ & $24 / 16$ & $60 / 40$ \\
\hline \multicolumn{5}{|l|}{ Drug usage } \\
\hline Yes/no & $13 / 27$ & $32.5 / 67.5$ & $32 / 8$ & $80 / 20$ \\
\hline \multicolumn{5}{|c|}{ Musculoskeletal pain } \\
\hline Yes/no & $13 / 27$ & $32.5 / 67.5$ & $19 / 21$ & $47.5 / 52.5$ \\
\hline
\end{tabular}

Sit and reach test The test is a common measure of flexibility and specifically measures the flexibility of the lower back and hamstring muscles. The most logical measure is to use the level of the feet as recording zero, so that any measure that does not reach the toes is negative and any reach past the toes is positive. However, using negative values is more difficult for statistical analyses and for comparing results. The procedure for the Presidents Challenge version require that the box is made with $23 \mathrm{~cm}$ at the level of the feet, so reaching $2 \mathrm{~cm}$ past the toes is recorded as $25 \mathrm{~cm}$. We used a table especially designed for this test. This test involved sitting on the floor with legs stretched out straight ahead. Shoes were removed. The soles of the feet were placed flat against the box. Both knees were locked and pressed flat to the floor. With the palms facing downward and the hands on top of each other or side by side, the participants reached forward along the measuring line as far as possible. We ensured that the hands remained at the same level, not one reaching further forward than the other. After some practice reaches, the participants reached out and held that position for at 1 to $2 \mathrm{~s}$ while the distance was recorded. Special attention was paid for jerky movements. The score was recorded to the nearest centimeter as the distance reached by the hand. We used the level of the $23 \mathrm{~cm}$ before the feet as the zero mark [18, 19].

Trunk lateral flexion test This aims to measure trunk flexibility laterally. Each participant was asked to stand with his/her feet shoulder-width apart and his/her hands adjacent to the trunk. The point of third finger on calf was marked. Then the participant flexed his/her trunk laterally as much as he/she could not move their hand apart from the calf. The point that their third phalanx reached on calf was marked, and the distance between those two points was measured with a tape measure. The test was repeated on both right and left sides [19].
Table 2 Comparison of physical properties of the sample

$I$ regular walking group $(N=40)$, II inactive controls $(N=40)$

${ }^{a}$ Independent samples $t$ test

\begin{tabular}{llcccc}
\hline Physical properties & Group & Min-max & Mean \pm SD & $t$ & $p^{\text {a }}$ \\
\hline Age (years) & I & $46.00-70.00$ & $56.30 \pm 4.85$ & 0.977 & 0.332 \\
Height $(\mathrm{cm})$ & II & $46.00-69.00$ & $55.15 \pm 5.64$ & & \\
& I & $149.00-181.00$ & $167.07 \pm 8.18$ & 0.854 & 0.396 \\
Weight $(\mathrm{kg})$ & II & $153.00-178.00$ & $165.57 \pm 7.50$ & & \\
& I & $50.00-97.00$ & $73.27 \pm 11.67$ & -1.632 & 0.107 \\
BMI $\left(\mathrm{kg} / \mathrm{m}^{2}\right)$ & II & $45.00-98.00$ & $77.50 \pm 11.46$ & & \\
& I & $19.53-33.21$ & $26.25 \pm 3.04$ & -2.814 & 0.006 \\
Waist-hip ratio & II & $18.49-34.92$ & $28.15 \pm 2.97$ & & \\
\hline
\end{tabular}


Muscle endurance Muscle endurance was measured with half-squat test [14].

Half-squat test The participants were in standing position with their hands flexed on trunk and feet shoulder apart without their shoes in the beginning of the test. With the start command of physical therapist, the participants began to do half squats as quickly as they could. The number of half squats completed in $1 \mathrm{~min}$ by the participants was recorded. The participants were asked not to squat down so much and to keep their heel on the floor [14].

Coordination Walking around two cones test was used to assess coordination. All participants were asked to have a seat in a chair located between two cones, which were placed $1.8 \mathrm{~m}$ on either side of or $1.5 \mathrm{~m}$ behind the chair. With the start command, the participants rose from the chair, walked to their right, going to the inside and around the back of the cone (counterclockwise), and returned to a fully seated position. One trial consisted of two complete circuits. Performance time was recorded in units of $0.1 \mathrm{~s}[20]$.

\section{Cognitive functions}

We used the Turkish version of Mini-mental Test (MMT) which was first published by Folstein in 1975 for the assessment of cognitive functions [21, 22]. The MMT is a common test used by health professionals in epidemiologic and clinic studies in order to assess cognitive function of elderly people. The test consists of subtests measuring orientation, calculation, attention, language, and memory. Total score is 30 and cutoff value for Turkish version is $<24$ showing cognitive damage [22].

\section{Emotional status}

Emotional status of the participants was evaluated with the Beck Depression Inventory. This was first developed by Beck et al. in 1961 [23]. There are 21 categories; each of them has four options. Participants were asked to choose the suitable option that expressed their emotional status in the last 1-week period. Total score is 63 and cutoff value for Turkish version is 17 which exposes the need for help [24].

\section{Quality of life}

The Center of Disease Control Health Related Quality of Life-4 (CDC HRQOL-4), which is an easy way to measure sense of well-being, was used to assess quality of life. The tool was developed in USA $[25,26]$, and validity and reliability of Turkish version was done in previous studies [1]. The tool includes four questions: Question 1 focuses on self-rated health that has been found to be predictive of mortality. Questions 2 and 3 relate to recent physical and mental health symptoms. Question 4 provides a global measure of disability. In the first question, general health was defined as excellent, very good, good, fair, and poor. The number of days that subject felt bad and had difficulty to manage the daily living activities due to physical and psychological problems are recorded (“Appendix”).

\section{Statistical analysis}

Statistical analyses were performed using the Statistical Package for the Social Sciences (SPSS version 13.0). Descriptive statistics are given as number, percentage, and mean \pm standard deviation (SD). The comparison of the groups was made by using independent samples $t$ test and Mann-Whitney $U$ test statistical analysis methods. In order to find out which factors (i.e., education level, occupation, or walking) affect cognitive function and emotional status, the multivariate general linear model (MANCOVA) analysis was conducted. The $p<0.05$ level was used to denote statistical significance.

\section{Results}

\section{Participants}

The mean age of 40 participants in the walking group was $56.30 \pm 4.85$ years, and the mean age of inactive participants $(N=40)$ was $55.15 \pm 5.64$ years. The participants' demographics are illustrated in Table 1. The physical properties of the sample are compared in Table 2. There were no differences between the two groups in terms of age, height, and weight $(p>0.05)$. The BMI and waist-hip ratio scores were different when the two groups were compared $(p<0.05)$. The average BMI and waist-hip ratio values were higher in the inactive group. Table 3 shows the walking characteristics of regular walking group. The participants in this group had been walking for $8.02 \pm$ 3.73 years, $4.42 \pm 1.15$ times a week, and the duration per a day was $79.25 \pm 27.65 \mathrm{~min}$.

Table 3 Walking characteristics of regular walking group

\begin{tabular}{lrrc}
\hline Walking characteristics & Min & Max & Mean \pm SD \\
\hline Walking period (years) & 3 & 20 & $8.02 \pm 3.78$ \\
Number of walking per a week (days) & 3 & 7 & $4.42 \pm 1.15$ \\
Duration of walking per a day (min) & 45 & 150 & $79.25 \pm 27.65$ \\
\hline
\end{tabular}


Table 4 Comparison of physical functions results of the sample

\begin{tabular}{llcl}
\hline Physical functions & Group & Mean \pm SD & $p$ \\
\hline Handgrip test & I & $66.27 \pm 21.68$ & $0.156^{\mathrm{a}}$ \\
& II & $59.32 \pm 21.68$ & \\
One-leg stand test & I & $125.50 \pm 46.97$ & $0.000^{\mathrm{a}}$ \\
& II & $83.27 \pm 38.13$ & \\
Sit-stand test & I & $10.36 \pm 1.81$ & $0.000^{\mathrm{a}}$ \\
& II & $13.28 \pm 1.76$ & \\
6 min walking test & I & $336.85 \pm 36.51$ & $0.000^{\mathrm{a}}$ \\
& II & $258.27 \pm 36.80$ & \\
Sit and reach & I & $25.18 \pm 6.46$ & $0.000^{\mathrm{b}}$ \\
& II & $20.40 \pm 2.77$ & \\
Trunk lateral flexion (R) & I & $19.65 \pm 3.40$ & $0.000^{\mathrm{a}}$ \\
& II & $13.67 \pm 2.58$ & \\
Trunk lateral flexion (L) & I & $20.02 \pm 3.48$ & $0.000^{\mathrm{a}}$ \\
& II & $14.10 \pm 2.21$ & \\
Half-squat & I & $50.57 \pm 7.93$ & $0.000^{\mathrm{a}}$ \\
Walking around two cones & II & $40.20 \pm 4.95$ & \\
& II & $6.76 \pm 0.62$ & $0.000^{\mathrm{b}}$ \\
\end{tabular}

$I$ regular walking group $(N=40), I I$ inactive controls $(N=40)$

${ }^{\text {a } I n d e p e n d e n t ~ s a m p l e s ~} t$ test

${ }^{\mathrm{b}}$ Mann-Whitney $U$ test

\section{Outcome measures}

Analysis of the data obtained from his study showed that there were significant differences in terms of physical functioning (Table 4), cognitive functioning (Table 5), emotional status (Table 6), and quality of life (Fig. 1; Table 7) between the groups $(p<0.05)$. The participants in the regular walking group had better scores in all parameters compared to the inactive controls. Namely, scores of muscle strength, balance, flexibility, cardiovascular and muscular endurance, and coordination of participants in the regular walking group were better. As well as the physical functioning, the scores of cognitive functioning, emotional status, and quality of life assessments were all better in the regular walking group. Moreover, nobody in inactive controls defined their general health as "excellent" or "very good" (Fig. 1). Although nobody in the sample showed depressive symptoms or cognitive impairments, the scores in terms of emotional status were found to be higher in the inactive controls. These results may show that keeping walking regularly have positive effects on cognitive function and emotional status, however (see details in Table 8). We found that education level and occupation had no effect on the cognitive function and emotional status of the participants. It was walking regularly that caused the difference between the two groups (Wilk's lambda=0.10).

\section{Discussion}

Advancing age is generally accompanied by a progressive decline in physical activity level. Age-related decline has been documented for functional fitness, including muscle strength, flexibility, balance, agility, gait velocity, and cardiorespiratory fitness. Walking has been considered to be the preferred choice of exercise among the European population [27, 28], probably because it is an accessible form of exercise that requires little expenditure, increases their individual choice in pace-setting, and has fewer risks than other forms of exercise. Participation in regular physical activity is associated with a reduced risk of many chronic illnesses and to provide many mental health benefits [3-5, 7]. The current minimum exercise recommendation associated with health benefit for each adult is to accumulate at least $30 \mathrm{~min}$ of moderate exercise on most days of the week $[4,6]$. Walking is a very acceptable form of exercise to a wide population. It does not require any formal training or special equipment and can be performed in an individual's locality and time [7, 8, 29]. The favorable effects of walking on both physiological and psychological well-being are firmly established [30]. Moreover, many positive effects are reported for primary and secondary prevention of disease, plus additional psychosocial benefits [31]. Concordant with these scientific evidence, we observed that there was a growing interest on physical activity especially walking among Turkish middle-aged and older people in our country and in city of Denizli as well. Our study was planned to examine the physical, cognitive functioning, and emotional status and quality of life in middle-aged and older adults attending to unsupervised regular walking program. The walking characteristics were defined according to aerobic exercise recommendations of American College of Sports Medicine and other authorities (at least three times a week, at least $45 \mathrm{~min}$ a day, and at least 1 year walking habit) [6].

When the participants in this study were compared in terms of occupation and education level, it was found that the participants in the regular walking group had higher education level and were consisted of retired officials. These results showed that education is an effective factor on health
Table 5 Comparison of cognitive functions of the sample

${ }^{\text {a }}$ Mann-Whitney $U$ test

\begin{tabular}{llccc}
\hline Cognitive function test & Group & Median & IQR & $p^{\text {a }}$ \\
\hline Mini-mental & Regular walking group $(N=40)$ & 26.00 & 3.50 & 0.006 \\
& Inactive controls $(N=40)$ & 26.00 & 1.00 & \\
\hline
\end{tabular}


Table 6 Comparison of emotional status of the sample

${ }^{\mathrm{a}}$ Independent samples $t$ test

\begin{tabular}{llrr}
\hline Emotional status test & Group & Mean \pm SD & $p^{\text {a }}$ \\
\hline Beck Depression Inventory & Regular walking group $(N=40)$ & $7.45 \pm 3.52$ & 0.000 \\
& Inactive controls $(N=40)$ & $13.20 \pm 3.97$ & \\
\hline
\end{tabular}

promotion. Another finding that attracted our attention was the number of male participants was higher than the female participants in the walking group. That means men prone to do regular exercise and to go out to participate in social activities. The physical properties of participants in the sample were similar. This shows us the homogeneity between groups. This also made the study strengthen. Another notable difference in terms of BMI and waist-hip ratio scores was found. The two scores were seen to be lower in walking group. This was an expected finding. It is well-known that regular exercise results in weight lost by increasing energy consumption and causing fat tissue destruction. A study searching the effects of different types of walking on body composition also supports that result [32].

We demonstrated that it is possible for community-living middle-aged and older adults to make significant gains attending in unsupervised regular walking program. All physical function parameters except handgrip improved in the participants of the unsupervised regular walking group. When the handgrip strength of participants was examined, it was seen that there was not any difference between the two groups of this study. This has also been reported in previous study by Wong et al. [13]. The authors examined the effects of physical activity level on physical performance and physical fitness of 123 participants who were 50 years and older. They measured handgrip strength of the participants, and then they did not found any relationship

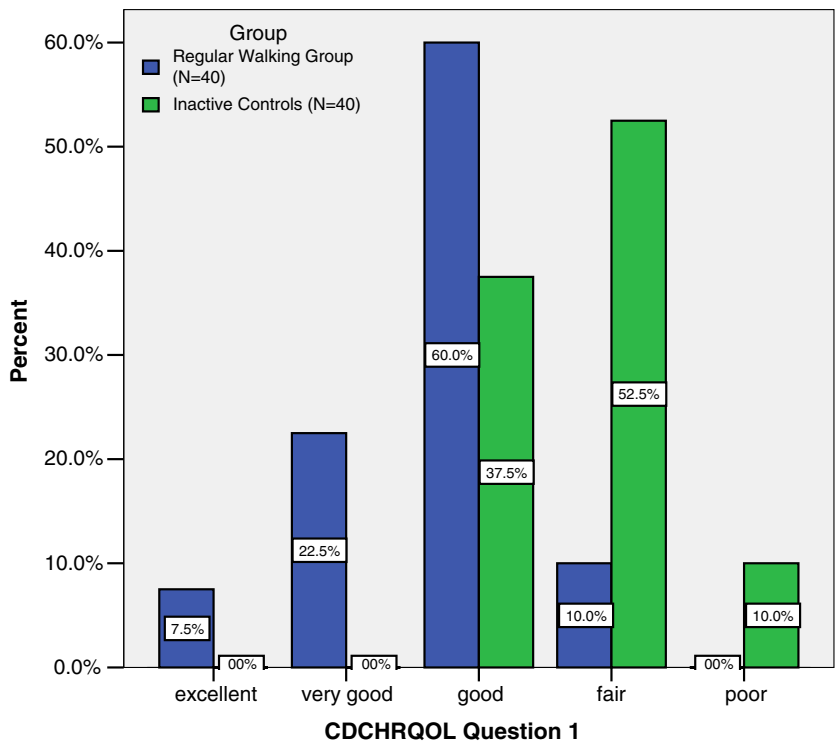

Fig. 1 Distribution of quality of life scale results (question 1) between activity level and handgrip strength. This and our study explain why walking as a physical activity has no effect on upper extremities function.

Loss of balance or postural control is most noticeable during the sixth decade of life. Impaired postural balance increases the risk of falls and is often a result of inactivity related decline in lower body musculoskeletal function. A recent report suggests possible improvement in postural stability in physically active men and women. In the results of another study, it was shown that physically active elderly performed significantly better on the static and dynamic balance tests than their inactive counterparts [15]. The results of our study also showed that regular walking as a dynamic aerobic exercise had positive effects on structures and ability of balance of the sample. Nakamura et al. [20] found that an exercise frequency of at least three times each week was effective on functional fitness in older adult women. The researchers found that participants who exercised three times a week showed most significant difference in the scores of walking around two cones test. We used the same test to evaluate coordination as mentioned in "Methods" section. The results of our study showed that the coordination of participants in the regular walking group were better than the inactive controls.

Gains attending in unsupervised walking program in cardiovascular fitness level were the poorest compared with the few similar studies in the related literature, especially in Turkey. This study also supports unsupervised walking program as a means of improving cardiovascular fitness. In our study, the 6-min test score was seen to be higher in the regular walking group. In some previous studies, it has been concluded that attendance to regular aerobic activities had two major effects beside psychosocial effects: (1) increased energy consumption and (2) increased cardiovascular fitness decreasing disease risk. In a meta-analysis, it was found that regular walking increased $0.6-6.9 \mathrm{ml} \mathrm{kg}^{-1}$

Table 7 Comparison of quality of life scale results of the sample

\begin{tabular}{llrl}
\hline CDC HRQOL-4 survey & Group & Mean \pm SD & $p^{\mathrm{a}}$ \\
\hline Question 2 (days) & I & $1.1 \pm 2.67$ & 0.000 \\
& II & $5.65 \pm 4.77$ & \\
Question 3 (days) & I & $1.07 \pm 1.65$ & 0.000 \\
& II & $6.15 \pm 7.15$ & \\
Question 4 (days) & I & $0.5 \pm 0.31$ & 0.000 \\
& II & $2.4 \pm 3.28$ & \\
\hline
\end{tabular}

${ }^{\mathrm{a}}$ Independent samples $t$ test

$I$ regular walking group $(N=40), I I$ inactive controls $(N=40)$ 
Table 8 The comparison of MMTS and BDS scores by MANCOVA

\begin{tabular}{lllll}
\hline Group & $\begin{array}{l}\text { MMTS } \\
\text { Mean } \pm \text { SD }\end{array}$ & $\begin{array}{l}\text { BDS } \\
\text { Mean } \pm \text { SD }\end{array}$ & $F$ & $p^{\mathrm{a}}$ \\
\hline $\begin{array}{c}\text { Regular walking } \\
\text { group }(N=40)\end{array}$ & $26.77 \pm 1.81$ & $7.45 \pm 3.52$ & 15.19 & $<0.05$ \\
$\begin{array}{c}\text { Inactive controls } \\
(N=40)\end{array}$ & $25.67 \pm 1.07$ & $13.20 \pm 3.97$ & 5.18 & $<0.05$ \\
\hline
\end{tabular}

MMTS Mini-mental Test Scale, BDS Beck Depression Inventory

${ }^{a}$ MANCOVA controlled factor analysis

in maximal oxygen consumption [32]. Although the results of some studies show that walking is not effective factor to increase cardiovascular fitness level, there have been many studies, including our study support that walking program can increase cardiovascular fitness of middle-aged and older people [20, 32, 33].

There were some notable differences between unsupervised walking group and inactive controls. Flexibility component of the unsupervised walking group was seen to be more improved. Teoman et al. [33] also reported significant difference in the flexibility of elderly women with exercise program (i.e., three times a week for 6 weeks) [12]. The more improved flexibility for the unsupervised walking group may reflect the physical gain of regular walking program.

It is a known fact that the oxidative capacity of muscle, which is very important for muscle strength and endurance, decreases with aging. Although the effect of walking on especially lower extremity strength and endurance is controversial, it was shown that regular aerobic exercise could increase mitochondria enzyme activity and $\mathrm{VO}_{2 \max }$ of muscle by increasing oxidative capacity [34]. The regular walking group had better lower extremity muscle endurance than inactive participants in our study. Advancing age is generally accompanied by a progressive decline in physical activity. Age-related decline has been documented for functional fitness including muscular strength and endurance, flexibility, balance, coordination, and cardiorespiratory fitness. For years, decline in these areas was thought to be a normal and necessary consequence of aging. Our study, supported by literature, indicates that decline relates more to regular physical activity and exercise levels than age, and the physical functions of middle-aged and older adults who walk regularly are better than inactive ones.

One aspect of function that has been well established as demonstrating age-related declines is cognitive function, typically characterized by decrements in a variety of processes including aspects of memory, attention, and perception. As a modifiable risk factor, physical inactivity has been implicated in depression and cognitive decline. Promoting physical activity is an effective strategy for the maintenance of cognitive function and brain plasticity in late life. There are many clinical, experimental, and radiologic studies about the effect of exercise on cognitive functions $[2,35,36]$. Early studies regarding the relationship between physical activity and cognition, which date back at least four decades, compared the cognitive performance of low and high fit adults on an array of paper and pencil computer-based tasks. In general, these studies found that higher fit individuals were able to perform more quickly and accurately on a wide variety of perceptual, cognitive, and motor tasks than low fit individuals. In the results of these studies, a clear and significant effect of aerobic exercise was found especially on tasks that involved executive control (i.e., planning, scheduling, and working memory), and exercise effects on cognition were found to be the largest for exercise training interventions that exceeded $30 \mathrm{~min}$ per session. Also, in experimental studies, physical activity has been found to increase cell proliferation, cell survival, and neurogenesis in the hippocampus. Physical activity has also been found to increase levels of nerve growth factors such as brain-derived neurotrophic factor which serves to enhance synaptic efficiency. In the PET and functional MRI studies, older adults with greater levels of aerobic fitness demonstrated significantly less gray matter loss in the frontal, temporal, and parietal lobes and significantly less tissue loss in the anterior and posterior white matter tracts [35-37]. Our results support the results showing that cognitive function of the middleaged and older adults walking regularly was better than the inactive controls.

Physical activity and exercise have been recommended as a treatment method for the successfully management of depression. The studies about the relationship between physical activity and mental health had been started since twentieth century and a lot of study was made recently. In the meetings Physical Activity, Physical Fitness and Health in France in 1992 and Surgeon's Report in USA, it was suggested that regular physical activity decreased mild level of depression and physical activity had benefits on preventing depression regardless of any relationship with age, sex, nationality, or socioeconomic levels. Reviews of the literature on the relationship between physical activity and mental health are in broad agreement that two are positively associated [3, 36-38]. In the study by Kramer et al., 8 weeks of regular, long bouts of walking significantly enhance feelings of vigor and activity and significantly reduce feelings of tension and anxiety compared inactive participants [35]. The results obtained from our study also showed that regular walking supported positively emotional status of middle-aged and older participants.

There is considerable and growing evidence that physical activity and/or exercise behavior plays a role in a person's perception of quality of life [4, 8, 27]. Sedentary lifestyle increase the risk factors for HT, DM, obesity, cancer, musculoskeletal diseases, and many other diseases $[6,7,9,31,39,40]$. The relationship between physical 
activity level and the decrease in the risk factors of these diseases has been reported in the literature [41-44]. Small but meaningful improvement in QoL can be brought about by exercise interventions over 3-6 months in well populations. Greater improvements were reported for QoL in response to moderate rather than light or vigorous exercise. However, there are few studies about the relationship between walking and quality of life. The result of our study, which shows that regular walking improves quality of life, contributes the literature in terms of relationship between physical activity, exercise, and quality of life. The more positive finding for the middle-aged and older adults walking regularly just evaluated in this study may reflect the increased social well-being and a good physical performance. This study has clearly demonstrated that even unsupervised regular walking produces changes in several aspect of health status. Moreover, this is feasible and can be suggested individuals over 45 years.

There was a limitation to the study. It was not possible to widen the sample size. Although we interviewed more than 200 potential participants, only 80 individuals who met inclusion criteria included. Most of them did not accept the invitation because of having no time to be tested.

\section{Conclusion}

It is known that physical activity and exercise decrease the risk factors related with cardiovascular diseases, disability, physical fitness level, and depression and improve quality of life. Levels of physical activity can be increased and the increase can be maintained. Interventions that encourage walking and do not require attendance at a facility are most likely to lead sustainable increases in physical activity. Walking has the greatest potential for increasing the overall activity levels of a sedentary population and meeting current health recommendations. It is the kind of exercise most likely to be adopted by a range of ages, socioeconomic and ethnic groups as well as both sexes. It is also associated with a lower risk of injury rates rather than other forms of exercise, and there are fewer barriers to walking than other types of physical activity. The results show that regular walking has positive effects on physical and mental health and quality of life in the aging period. Therefore, health professionals should recommend and encourage middle-aged and older adults to walk regularly, at least 30-45 min, three times a week. The study emphasized that more open-ended activities such as regular walking may be more appropriate for middle-aged and older adults. The authors also recommend that local authorities and governments should support healthy lifestyle and provide suitable environment and encourage people to make regular walking in their daily life and accept this as a health policy.

\section{Appendix}

Table 9 Questions of the centers of disease control health related quality of life-4 survey

1. Self-perceived health
Would you say that in general your health is?
Excellent
Very good
Good
Fair
Poor
2. Recent physical health
Now thinking about your physical health, which includes physical
illness and injury, for how many days during the past 30 days
was your physical health not good?
3. Recent mental health
Now thinking about your mental health, which includes stress,
depression, and problems with emotions, for how many days
during the past 30 days was your mental health not good?
4. Recent activity limitation
During the past 30 days for about how many days did poor physical
or mental health keep you from doing your usual activities, such
as self-care, work, or recreation?

\section{References}

1. Cavlak U, Yagci N et al (2009) A new tool measuring heathrelated quality of life (HRQOL): the effects of musculoskeletal pain in a group of older Turkish people. Arch Gerontol Geriatr 49 (2):298-303

2. Laukkanen JA, Laaksonen D et al (2009) Determinants of cardiorespiratory fitness in men aged 42 to 60 years with and without cardiovascular disease. Am J Cardiol 103:1598-1604

3. United Kingdom Department of Health, Physical Activity, Health Improvement and Prevention (2004) At least five a week: evidence on the impact of physical activity and its relationship to health, a report from the Chief Medical Officer. United Kingdom Department of Health, Physical Activity, Health Improvement and Prevention, London

4. World Health Organization (2003) Annual global move for health initiative: a concept paper. World Health Organization, Geneva

5. United States Department of Health and Human Services (1996) Physical activity and health: a report of the Surgeon General. US Department of Health and Human Services, Centers for Disease Control and Prevention, National Center for Chronic Disease Prevention and Health Promotion, Atlanta

6. American College of Sports Medicine (2006) ACSM's guidelines for exercise testing and prescription, 7th edn. Lippincott, Williams, \& Wilkins, Philadelphia, p 123

7. Davies KM, Hare JSJ, Morris CL (2009) The impact of walking on health: a literature review. J Health Social Care Improv 1(4):1-11

8. Ekkekakis P, Backhouse SH et al (2007) Walking is popular among adults but is it pleasant? A framework for clarifying the link between walking and affect as illustrated in two studies. Psychol Sport Exerc 9:246-264

9. Hakim AA, Petrovitch $\mathrm{H}$ et al (1998) Effects of walking on mortality among nonsmoking retired men. New England Journal of Medicine 338:94-99 
10. Tsuji I, Takahashi K et al (2003) Impact of walking upon medical care expenditure in Japan: the Ohsaki Cohort Study. International Journal of Epidemiology 32:809-814

11. Eyigor S, Karapolat $\mathrm{H}$ et al (2009) A randomized controlled trial of Turkish folklore dance on the physical performance, balance, depression and quality of life in older women. Arch Gerontol Geriatr 48:84-88

12. Tsang RCC (2005) Reference values for 6-minute walk test and hand-grip strength in healthy Hong Kong Chinese adults. Hong Kong Physiotherapy Journal 23:6-12

13. Wong CH, Wong SF et al (2003) Habitual walking and its correlation to better physical function: implications for prevention of physical disability in older persons. J Gerontol 58:555-560

14. Baskan E, Cavlak U, Telli O (2006) The effect of maximal isometric contraction training in various knee positions on physical capacity of healthy quadriceps muscle. Sports Medicine Journal 8:464-469

15. Bulbulian R, Hargan ML (2000) The effect of activity history and current activity on static and dynamic postural balance in older adults. Physiol Behav 70:319-325

16. Colakoglu FF (2008) The effect of callisthenic exercise on physical fitness values of sedentary women. Science \& Sports 23:306-309

17. Saad HB, Prefaut C et al (2009) 6-Minute walk distance in healthy North Africans older than 40 years: influence of parity. Respiratory Medicine 103:74-84

18. Wells KF, Dillon EK (1952) The sit and reach. A test of back and leg flexibility. Research Quarterly 23:115-118

19. Bas U (1998) The effects of Hatha yoga and classic exercise on postural and physical fitness properties of healthy young participants. Master of science thesis, Hacettepe University, Institute Of Health Sciences, Ankara

20. Nakamura Y, Tanaka K et al (2007) Effects of exercise frequency on functional fitness in older adult women. Arch Gerontol Geriatr 44:163-173

21. Folstein MF, Folstein SE, McHugh PR (1975) Mini-mental state. A practical method for grading the cognitive state of patients for the clinician. J Psychiatr Res 12(3):189-198

22. Güngen C, Ertan T et al (2002) The validity and reliability of standardized mini mental in Turkish population. Turkish Journal of Psychiatry 13(4):273-281

23. Beck AT, Ward CH et al (1961) An inventory for measuring depression. Arch Gen Psychiatry 4:561-571

24. Hisli N (1988) A study about validity of Beck Depression Inventory. J Psychology 6(22):118-122

25. Mobiliy PR, Herr KA et al (1994) An epidemiologic analysis of pain in elderly the Iowa 65+ rural health study. J Aging Heart 6:143-154

26. Morriarity DG, Kobau R et al (2005) Tracking healthy days - a window on health of older adults. Prev Choronic Dis 2:A16

27. Dunn AL, Garcia ME et al (1998) Six-month physical activity and fitness changes in project active, a randomized trial. Medical and Science in Sports and Exercise 20:1076-1083
28. Vaz De Almeida MD, Graca P et al (1999) Physical activity levels and body weight in a nationally representative sample in the European Union. Public Health Nutrition 2:105-113

29. Lee L, Avis M, Arthur A (2007) The role of self-efficacy in older people's decisions to initiate and maintain regular walking as exercise - findings from a qualitative study. Preventive Medicine 45:62-65

30. Garatachea N, Ov M et al (2008) Feelings of well being in elderly people: relationship to physical activity and physical function. Arch Gerontol Geriatr 48:306-312

31. Castillo G, Ortega PFB, Ruiz J (2005) Improvement of physical fitness as anti-aging intervention. Med Clin (Barc) 124(4):146155

32. Murtagh EM, Boreham C et al (2005) The effects of 60 minutes of brisk walking per week, accumulated in two different patterns, on cardiovascular risk. Preventive Medicine 41:92-97

33. Teoman N, Ozcan A et al (2004) The effect of exercise on physical fitness and quality of life in postmenopausal women. Maturitas 20(47(1)):71-77

34. Durheim MT, Slentz CA et al (2008) Relationships between exercise-induced reductions in thigh intermuscular adipose tissue, changes in lipoprotein particle size, and visceral adiposity. Am J Physiol Endocrinol Metab 295(2):407-412

35. Kramer AF, Colcombe SJ et al (2005) Fitness, aging and neurocognitive function. Neurobiology of Aging 26:124-127

36. Merom D, Phongsavan P et al (2008) Promoting walking as an adjunct intervention to group cognitive behavioral therapy for anxiety disorders - a pilot group randomized trial. Journal of Anxiety Disorders 22:959-968

37. Pontifex MB, Hillman CH (2008) Neuroelectric measurement of cognition during aerobic exercise. Methods 45(4):271-278

38. Jedrziewskia MK, Leea V, Trojanowskia JQ (2007) Physical activity and cognitive health. Alzheimer's \& Dementia 3:98-108

39. Sardinha LB, Teixeira PJ et al (2000) Subcutaneous central fat is associated with cardiovascular risk factors in men independently of total fatness and fitness. Metabolism 49(11):1379-1385

40. Harris A, Cronkite R, Moos R (2006) Physical activity, exercise coping, and depression in a 10-year cohort study of depressed patients. J Affect Disord 93:79-85

41. Osei-Tutu KB, Campagna PD (2005) The effects of short- vs long bout exercise on mood, $\mathrm{VO}_{2} \max$, and percent body fat. Preventive Medicine 40:92-98

42. Aslan BU, Cavlak U et al (2008) Balance performance, aging and falling: a comparative study based on a Turkish sample. Arch Gerontol Geriatr 46:283-292

43. Davis J, Murphy M et al (2007) Acute effects of walking on inflammatory and cardiovascular risk in sedentary post-menopausal women. Journal of sports Sciences 1:1-7

44. Murphy M, Nevill A et al (2007) The effect of walking on fitness, fatness and resting blood pressure: a meta-analysis of randomized, controlled trials. Preventive Medicine 44:377-385 